Artigo Original

\title{
Efeitos da participação em um Grupo de Coluna sobre as dores musculoesqueléticas, qualidade de vida e funcionalidade dos usuários de uma Unidade Básica de Saúde de Porto Alegre - Brasil
}

\author{
Raquel Gonçalves Borges \\ Adriane Vieira \\ Matias Noll \\ Patrícia Thurow Bartz \\ Cláudia Tarragô Candotti \\ Escola de Educação Física da Universidade Federal do Rio Grande do Sul, Porto Alegre,
RS, Brasil \\ Resumo: O objetivo deste estudo foi avaliar a eficácia do Grupo de Coluna ministrado em uma Unidade \\ Básica de Saúde de Porto Alegre. Foram avaliados e reavaliados 29 usuários, os quais participaram do \\ Grupo de Coluna. Foi utilizada a Escala Visual Analógica para avaliar a intensidade da dor, o questionário \\ Oswestry Disability Índex para avaliar a capacidade funcional, e o questionário Short-Form Health Survey - \\ SF-36 para avaliar a qualidade de vida. Os resultados, obtidos a partir do teste de Wilcoxon $(\alpha=0,05)$, \\ demonstraram a eficácia do Grupo de Coluna na melhora da intensidade das dores musculoesqueléticas \\ $(p<0,05)$, na capacidade funcional $(p=0,005)$, e na qualidade de vida nos domínios capacidade funcional \\ $(p=0,001)$, limitações por aspectos físico $(p=0,001)$, dor $(p=0,001)$, vitalidade $(p=0,004)$, limitação emocional \\ $(p=0,026)$ e saúde mental $(p=0,010)$. Conclui-se que o Grupo de Coluna foi eficaz na melhora da dor, \\ funcionalidade e qualidade de vida dos usuários.
}

Palavras-chave: Escola Postural. Funcionalidade. Qualidade de vida.

\section{Effects of participation in a Back School on musculoskeletal pain, quality of life and functionality of users of a Unidade Básica de Saúde from Porto Alegre-Brazil}

\begin{abstract}
This study was carried out in order to evaluate the effectiveness of the Back Group project, in a Porto Alegre's Basic Health Unit. We evaluated and re-evaluated 29 users before and after the participated in a Back Group. It's were used the Visual Analogue Scale to assess pain, the Oswestry Disability Index to assess the functionality, and the questionnaire Item Short-Form Health Survey-SF-36 to assess the quality of life. The results, obtained from the Wilcoxon test $(\alpha=0.05)$, demonstrated the effectiveness of the Back Group in the improvement of the intensity of musculoskeletal pain $(p<0.05)$, in the physical functioning $(p=0.005)$, in the quality of life in the functional capacity $(p=0.001)$, by the physical limitations $(p=0.001)$, pain $(p=0.001)$, vitality $(p=0.004)$, emotional limitation $(p=0.026)$ and mental health $(p=0.010)$ dominions. It is concluded that the Back Group was effectiveness in the improved of the pain, function and quality of life's users.
\end{abstract}

Key Words: Back School. Functionality. Quality of life.

\section{Introdução}

O desconforto gerado pelas dores crônicas interfere na qualidade de vida de diversas pessoas, prejudicando a realização de atividades de vida diária ( musculoesqueléticas são problemas freqüentes $\mathrm{e}$ podem ter causas múltiplas, estando, muitas vezes, relacionadas a variáveis psicossociais e ambientais, assim como a condições precárias de vida e saúde, à falta de informações e ao uso incorreto da mecânica corporal no trabalho e no domicílio (ALMEIDA et al., 1999; BRINGUENTE et al., 1997; KNOPLICH, 2003).

Vários autores referem-se à alta incidência e prevalência de dores nas costas, afirmando que cerca de $80 \%$ da população já experimentou, em algum momento da vida, queixas de dores na coluna (CHUNG, 1996; DEYO et al., 1991; FRYMOYER; CATSBARIL, 1991; SCHMIDT; KOHLMANN, 2005). As queixas de dor, assim como sua intensidade, são relevantes e podem interferir significativamente na qualidade de vida dos indivíduos, gerando problemas psicológicos, mudanças de comportamento, redução da capacidade física, diminuindo a produtividade nas tarefas de casa e do trabalho (OLIVEIRA et al., 2004).

A capacidade funcional está relacionada à autonomia e à independência do sujeito na realização de atividades diárias (GRIMLEY,1984). 
Para Rosa et al (2003) a incapacidade funcional define-se pela presença de dificuldade ou impossibilidade no desempenho de certos gestos e de certas atividades da vida cotidiana.

A qualidade de vida ainda é um campo em debate. Fleck et al (2008) comentam que a definição proposta pela a Organização Mundial da Saúde é a que melhor traduz a abrangência do construto qualidade de vida, definindo-o como a percepção do indivíduo em relação à sua posição na vida, de um completo bem estar físico, mental e social. O conceito "qualidade de vida" incorpora de forma complexa a saúde física, o estado psicológico, o nível de independência, as relações sociais, as crenças pessoais e a relação com aspectos significativos do meio ambiente (FLECK et al., 2008).

A qualidade de vida inclui, portanto, aspectos relacionados com a saúde, o bem-estar físico, funcional, emocional e mental, mas também fatores sociais como 0 relacionamento com amigos, familiares e colegas de trabalho (TSUKIMOTO et al., 2006). Nas últimas décadas, surgiram várias pesquisas com diferentes abordagens $\mathrm{e}$ instrumentos para avaliar a qualidade de vida (MAZO, 2008), com objetivo de sistematizar uma avaliação da qualidade de vida, funcionalidade e percepção do quadro álgico e do estado de saúde.

Para a melhora da qualidade de vida das populações, a educação em saúde é uma das estratégias que vem sendo incentivadas nas políticas públicas (BRASIL, 2006). Ações educativas são estimuladas, especialmente, nos serviços de atenção básica à saúde através de grupos que abordam hábitos e estilos de vida, nestes casos, os programas ou ações tendem a concentrar-se em componentes relacionados a riscos comportamentais passíveis de mudanças, que estariam, pelo menos em parte, sob 0 controle dos próprios indivíduos (BUSS, 2000).

Dentre os recursos utilizados para promover a saúde, ressalta-se a relevância das Escolas Posturais, as quais visam à redução de dores musculoesqueléticas crônicas, a partir do ensino da adequada execução das Atividades de Vida Diária (AVD's), evitando a diminuição da funcionalidade e na qualidade de vida dos participantes (FERREIRA; NAVEGA, 2010), visto que os hábitos posturais inadequados são um fator desencadeante de tensões musculares, por conseqüência, de quadros álgicos que interferem na qualidade de vida e na funcionalidade corporal e que podem ser modificados a partir de uma intervenção educativa (

Souza e Vieira (2003) comentam que a Escola Postural visa não apenas amenizar as disfunções da coluna, mas também conscientizar a população atendida e assim facilitar a aquisição de hábitos posturais mais saudáveis, principalmente nas AVD's.

A habilidade de executar as AVD's com facilidade implica um melhor conhecimento sobre o próprio movimento, ou seja, um melhor conhecimento da dinâmica de movimento durante a execução de AVD's (VIEIRA, 2006).

Deste modo, os programas de Escola Postural, normalmente ministrados em serviços de atenção secundária e direcionados para pacientes com dor lombar crônica não específica (ANDRADE et al., 2005), têm servido de referência para formação de Grupos de Coluna em Unidades Básicas de Saúde (UBS).

$\mathrm{Na}$ Universidade Federal do Rio Grande do Sul (UFRGS), a inserção de estudantes no serviço de atenção básica à saúde motivou a implantação do projeto de extensão "Grupo de Coluna no contexto do SUS" em 2009, o qual, desde o início, está estruturado em cinco encontros de duas horas. Os encontros são ministrados por uma professora fisioterapeuta e estudantes de graduação dos cursos de Fisioterapia e Educação Física. Os encontros são teórico-práticos e tomam por referência a Escola Postural Sueca (FORSSELL, 1980), cujo os objetivos são auxiliar os participantes a entenderem a estrutura $e$ funcionalidade da coluna vertebral, orientar a realização de AVD's e propor exercícios básicos para melhora da postura. $O$ projeto prevê avaliações realizadas antes de iniciar o Grupo e após seu término. O público-alvo são usuários acima de 30 anos que apresentam dores musculoesqueléticas e que, por indicação dos médicos da UBS estejam inscritos na lista de espera para participar do Grupo de Coluna. Tendo em vista a realidade do serviço, que enfatiza a atenção básica à saúde e atende a uma população adscrita a um território determinado de Porto Alegre, o Grupo de Coluna, apesar de enfatizar os cuidados com a coluna lombar, acolhe todos os usuários encaminhados ao Grupo por apresentarem queixas álgicas no sistema musculoesquelético. 
Assim, justifica-se o presente estudo, o qual objetiva avaliar a eficácia do projeto de extensão Grupo de Coluna no contexto do SUS ministrado para usuários de uma UBS de Porto Alegre. Este estudo visa identificar se esse programa de educação em saúde contribui para a promoção da saúde e a prevenção de agravos, amenizando as dores musculoesqueléticas e ampliando a qualidade de vida e a funcionalidade dos usuários de uma UBS de Porto Alegre.

\section{Materiais e Método}

Este estudo caracteriza-se como de tipo semiexperimental e utiliza métodos quantitativos de análise (GAYA et al., 2008). A utilização de métodos quantitativos é relevante para a avaliação de resultados no quadro de intensidade das queixas de dor, funcionalidade e qualidade de vida dos participantes. A opção por um estudo sem grupo controle deve-se a realidade do projeto de extensão e do serviço onde é realizado o projeto.

A amostra foi composta por participantes do projeto de extensão "Grupo de Coluna no contexto do SUS" desenvolvido numa UBS de Porto Alegre. Os critérios de seleção para participar do projeto de pesquisa foram: idade igual ou superior a 30 anos, dor musculoesquelética há mais de três meses e encaminhamento pelos médicos da UBS.

Foram avaliados (pré-teste) 47 usuários $(57,7 \pm 11,8$ anos) uma semana antes de começar o Grupo de Coluna. Destes 47 usuários avaliados inicialmente, $29(55,9 \pm 11,4$ anos) participaram do Grupo de Coluna, sendo reavaliadas (pós-teste) após finalização do Grupo, e 18 (59,50 13,8 anos) pessoas não aderiram ao programa após serem avaliadas.

A Tabela 1 apresenta as características de todos os usuários avaliados, e dos usuários que aderiram (AG) e não aderiram (NAG) ao Grupo de Coluna.

A avaliação da dor foi realizada a partir da Escala Visual Analógica (EVA), que avalia a intensidade de dor, e do questionário Oswestry Disability Índex (ODI), que avalia a limitação funcional imposta pela dor.

A EVA consiste em auxiliar na medição da intensidade da dor do paciente, questionando-o quanto ao seu grau de dor. É constituída por uma linha reta e horizontal de $10 \mathrm{~cm}$, onde 0 ponto inicial corresponde à ausência de dor e o ponto final da linha ao nível mais insuportável de dor sentida pelo indivíduo (CARAVIELLO et al., 2005). A EVA é um instrumento importante para verificar a percepção de incômodo da dor e para analisar se a intervenção direcionada à redução da dor está sendo efetiva. Neste estudo, as queixas álgicas foram agrupadas em membros superiores, membros inferiores e coluna.

Os usuários do Grupo de Coluna identificaram, durante a anamnese, até seis locais onde apresentavam dores musculoesqueléticas. Após a identificação do local, eles próprios marcaram na escala a intensidade de cada local de dor identificado. Dentre os usuários avaliados que aderiram ao Grupo, houve 27 queixas de dores na coluna, $13 \mathrm{em}$ membros inferiores e $15 \mathrm{em}$ membros superiores.

Tabela 1. Características de todos os usuários avaliados e dos que aderiram (AG) e os que não aderiram (NAG) ao Grupo de Coluna.

\begin{tabular}{ccccccc} 
& \multicolumn{2}{c}{ Total } & \multicolumn{2}{c}{ AG } & \multicolumn{2}{c}{ NAG } \\
\cline { 2 - 7 } & $\mathrm{n}$ & $\%$ & $\mathrm{~N}$ & $\%$ & $\mathrm{~N}$ & $\%$ \\
\hline Sexo & & & & & & \\
Masculino & 8 & 17 & 8 & 72,4 & 0 & 0 \\
Feminino & 39 & 83 & 21 & 27,6 & 18 & 100 \\
Total & 47 & 100 & 29 & 100 & 18 & 100 \\
\hline Idade (anos) & & & & & & \\
$\leq 49$ & 11 & 23,4 & 7 & 24,1 & 4 & 22,2 \\
$50-59$ & 14 & 29,8 & 9 & 31 & 5 & 27,7 \\
$60-69$ & 12 & 25,5 & 10 & 34,5 & 2 & 11,1 \\
$\geq 70$ & 10 & 21,3 & 3 & 10,3 & 7 & 38,8 \\
Total & 47 & 100 & 29 & 100 & 18 & 100 \\
\hline
\end{tabular}

O questionário ODI objetiva avaliar as limitações funcionais em indivíduos que apresentam dores musculoesqueléticas por meio da análise de AVD's, visando identificar o impacto da dor crônica no cotidiano desses indivíduos (FAIRBANK et al., 1980; FAIRBANK, 2000).

O ODI, adaptado e validado para a língua portuguesa em 2006 (VIGATTO et al., 2007), apresenta dez seções, sendo a primeira a intensidade de dor. As demais seções verificam as limitações impostas pela dor na realização das seguintes atividades cotidianas: proporcionar cuidado pessoal, levantar objetos, andar, permanecer sentado e em pé, dormir, vida sexual e locomoção (VIGATTO, 2006). Os resultados são classificados da seguinte forma: Incapacidade Mínima (0\% a 20\%), Incapacidade Moderada (20\% a $40 \%)$, Incapacidade Grave $(40 \%$ a $60 \%)$, "Geradora de Invalidez" (60\% a $80 \%$ ), Paciente Preso a cama (80\% a $100 \%)$ FAIRBANK et al., 1980; FAIRBANK, 2000). 
Os domínios do ODI variam de zero a 100 (cem), onde zero é a melhor e 100 é a pior na pontuação FAIRBANK et al., 1980; FAIRBANK, 2000).

Para avaliar a qualidade de vida dos participantes, foi utilizado o questionário Medical Outcomes Study 36 - Item short form health survey (SF-36), traduzido e validado para 0 português por Ciconelli (1998).

Martinez (2002) demonstra que o SF-36 é um questionário genérico, com conceitos não específicos e que permite comparações entre diferentes problemas de saúde e tratamentos. Considera a percepção dos indivíduos quanto ao seu próprio estado de saúde e contempla os aspectos mais representativos da saúde.

O SF-36 é um questionário que avalia a qualidade de vida e se suas medidas estão relacionadas com o funcionamento da saúde em oito dominios: capacidade funcional, aspectos físicos, dor, vitalidade, estado geral de saúde, aspectos sociais, limitações emocionais e saúde mental. O SF-36 faz os relatórios de qualidade de vida dos pacientes percebida por escores variando de zero a 100 (cem), onde 100 é a melhor e zero é a pior na pontuação (TAVAFIAN et al., 2007).

Este estudo foi apreciado e aprovado pelo Comitê de Ética em Pesquisa do Hospital de Clínicas de Porto Alegre ( $\left.n^{\circ} 100354\right)$ e garantiu o sigilo de dados pessoais e confidenciais, evitando constrangimentos ou prejuízos aos sujeitos de pesquisa, tornando anônimos os dados obtidos. Os dados serão guardados durante cinco anos, findo os quais serão destruídos conforme instruções da Resolução 196/96, IX.

Os instrumentos EVA, ODI e SF-36 foram utilizados, antes de iniciar (pré-teste) e após (pósteste) a realização do Grupo de Coluna. O registro da intensidade da dor e a aplicação dos questionários tiveram como objetivo documentar e comparar as percepções de dor, funcionalidade e qualidade de vida dos participantes nesses diferentes momentos. Os questionários foram também utilizados para comparar o perfil dos usuários que aderem e os que não aderem ao Grupo de Coluna. Todos os usuários responderam aos questionários, sendo que, por falhas no preenchimento, dois questionários do SF-36 e cinco do ODI não foram considerados na análise dos dados.

\section{Metodologia do Grupo de Coluna}

O Grupo de Coluna está estruturado em cinco encontros. Cada encontro tem duração de duas horas, sendo os primeiros 40 minutos direcionados ao conhecimento e à discussão de temas teóricos relacionados à postura, à estrutura corporal e à realização adequada de AVD's (Quadro 1). A parte prática tem duração de 1 hora e 10 minutos e inicia com a simulação de AVD's, atividades lúdicas e/ou auto-massagem. Posteriormente são realizados exercícios de fortalecimento, alongamento e relaxamento da musculatura do tronco e membros inferiores. Os encontros finalizam com uma breve massagem realizada pela equipe de trabalho.

\section{Análise estatística}

A análise estatística foi realizada no Software SPSS 18.0. Os valores obtidos pelos instrumentos de avaliação foram analisados por meio da estatística descritiva e inferencial. A análise estatística descritiva foi realizada a partir de médias e desvio-padrão da população e tabelas de freqüência.

A estatística inferencial objetivou comparar os resultados obtidos no pré e pós-teste do grupo AG. Para realização da estatística inferencial foi utilizado o teste de Wilcoxon (1) para verificar diferenças entre pré e pós-teste para as varáveis provenientes dos 8 domínios do SF-36 e do ODI; e (2) para verificar diferenças entre pré e pósteste para variável dor. $\mathrm{O}$ nível de significância adotado foi de 0,05 .

Quadro 1. Temas Teóricos dos Cinco Encontros do Grupo de Coluna.

1) Aspectos biológicos, culturais e psicológicos da postura corporal

2) Noções básicas sobre estrutura e funcionalidade da coluna vertebral e manutenção das posturas sentada e em pé

3) Relações entre movimentos da coluna e membros inferiores e a execução dos atos de sentar, agachar e carregar objetos

4) Posicionamento e realização de atividades na posição deitada, posturas ao dormir e ato de deitar e levantar da cama

5) Execução assistida e comentada de AVD's escolhidas pelos participantes

\section{Resultados}

Os dados obtidos a partir da EVA demonstraram, após a finalização dos 5 encontros, uma diminuição na intensidade das 
dores nos usuários que aderiram ao grupo em todos os segmentos corporais analisados: membros superiores, membros inferiores e coluna vertebral. Os dados obtidos a partir da EVA estão descritos na Tabela 2 .

Tabela 2. Média, desvio padrão e nível de significância para a variável intensidade da dor na avaliação e reavaliação para os usuários que aderiram ao Grupo de Coluna.

\begin{tabular}{ccccc} 
& & Pré-teste & Pós-Teste & Valor de $\boldsymbol{p}$ \\
\hline \multirow{2}{*}{ Segmentos } & Membros Inferiores $(n=13)$ & $5,78 \pm 3,00$ & $2,81 \pm 3,10$ & $0,001^{*}$ \\
corporais $(n=29)$ & Membros Superiores $(n=15)$ & $5,34 \pm 2,67$ & $1,93 \pm 3,28$ & $0,001^{*}$ \\
& Coluna Vertebral $(n=27)$ & $5,75 \pm 2,10$ & $2,80 \pm 2,04$ & $0,001^{*}$ \\
\hline${ }^{*} p<0,05$
\end{tabular}

Tabela 3. Média, desvio padrão e nível de significância do ODI na avaliação e reavaliação dos usuários que aderiram ao Grupo de Coluna.

\begin{tabular}{lccc}
\cline { 2 - 4 } & Pré-teste & Pós-teste & Valor de $\boldsymbol{p}$ \\
\hline ODI $(n=24)$ & $24,42 \pm 11,50$ & $17,75 \pm 10,01$ & $0,005^{\star}$ \\
\hline${ }^{*} p<0,05$ & & &
\end{tabular}

Na análise do ODI, observou-se também uma diferença significativa entre a avaliação e reavaliação, tendo a média do grupo passado de uma incapacidade moderada para uma incapacidade mínima (Tabela 3).
Os dados do questionário SF-36 demonstraram, na reavaliação, um aumento da média de todos os domínios, sendo essa diferença significativa nos domínios capacidade funcional, aspectos físicos, dor, vitalidade, limitações emocionais e saúde mental (Tabela 4).

Tabela 4. Média, desvio padrão e nível de significância para os oito domínios do questionário SF-36 na avaliação e reavaliação dos usuários que aderiram ao Grupo de Coluna.

\begin{tabular}{clccc} 
& & Pré-teste & Pós-teste & Valor de $\boldsymbol{p}$ \\
\cline { 3 - 4 } & Capacidade Funcional & $49,81 \pm 26,15$ & $65,55 \pm 15,77$ & $0,001^{*}$ \\
& Aspectos Físicos & $25,92 \pm 36,35$ & $56,48 \pm 37,08$ & $0,001^{*}$ \\
SF-36 & Dor & $34,44 \pm 14,57$ & $50,25 \pm 14,97$ & $0,001^{*}$ \\
(Domínios) & Estado Geral de Saúde & $50,03 \pm 11,53$ & $50,92 \pm 10,82$ & 0,798 \\
n=27 & Vitalidade & $50,92 \pm 13,45$ & $58,15 \pm 9,62$ & $0,004^{*}$ \\
& Aspectos Sociais & $64,81 \pm 27,31$ & $72,22 \pm 22,82$ & 0,166 \\
& Emocionais & $51,84 \pm 42,70$ & $64,19 \pm 38,04$ & $0,026^{*}$ \\
& Saúde Mental & $58,81 \pm 20,51$ & $66,78 \pm 20,18$ & $0,010^{*}$ \\
\hline${ }^{*} \mathrm{p}<0,05$ & & & &
\end{tabular}

\section{Discussão}

Este estudo buscou identificar se o Grupo de Coluna contribuiu para a promoção da saúde. Candeias (1997) comenta que a educação em saúde procura desencadear mudanças de comportamento individual, enquanto que a promoção em saúde, muito embora inclua a educação em saúde, visa a provocar mudanças de comportamento organizacional (estrutural) capazes de beneficiar a saúde de camadas mais amplas da população.

Os resultados do presente estudo demonstraram a influência positiva do Grupo de Coluna no Contexto do SUS, visto que o mesmo foi eficaz na diminuição da intensidade das dores musculoesqueléticase na melhora da qualidade de vida e da funcionalidade dos participantes.
As intensidades das dores musculoesqueléticas as quais foram separadas em membros superiores, membros inferiores e coluna vertebral, indicaram em seus resultados que as mesmas diminuíram após os encontros do Grupo de Coluna. No questionário ODI verificouse uma melhora significativa na capacidade funcional das pessoas que participaram do Grupo de Coluna.

Resultados similares foram encontrados por Caraviello et al, (2005) que avaliou a evolução da dor e as limitações funcionais dos participantes de um programa de Escola de Coluna. A EVA e o Questionário de Roland - Morris (RM) foram os instrumentos aplicados no início e após o término da Escola de Coluna. No final da Escola a maior parte dos pacientes apresentou melhora da dor 
$(56,7 \%)$ e da função (60\%) ( 2005).

Andrade et al (2008) avaliaram 70 pacientes com lombalgia crônica inespecífica num programa de Escola de Coluna por três meses, utilizando os instrumentos RM e EVA. A conclusão destes autores foi que o programa proposto de Escola de Coluna com conteúdo teórico-prático, influenciou na diminuição da intensidade de dor e aumento da capacidade funcional, com preservação dos benefícios na intensidade da dor e capacidade funcional por três meses, sendo uma opção segura e de baixo custo na prevenção e tratamento de pacientes com lombalgia crônica inespecífica.

Os autores Ferreira e Navega (2010) também utilizaram em seu estudo o questionário ODI. A amostra foi composta de 41 pessoas de ambos os sexos com idade entre 25-65 anos que participaram de um programa de seis encontros de uma hora semanal. Seus resultados demonstraram uma melhora significativa da capacidade funcional dos participantes após a finalização do programa.

Os resultados do questionário SF-36, no presente estudo, demonstraram que dentre os 8 domínios analisados, capacidade funcional, limitações por aspectos físicos, dor, vitalidade, limitações emocionais e saúde mental apresentaram melhoras significativas. Do mesmo modo, Tsukimoto et al (2006) em seu trabalho avaliou 110 pessoas que cumpriram integralmente um programa Escola de Postura, mediante a avaliação dos questionários "RolandMorris" (RM), "Short Form Health Survey (SF36) e da escala visual analógica (EVA). Seus resultados mostraram que os indivíduos que concluíram a Escola de Postura apresentaram melhora significativa em cinco domínios do SF-36: capacidade funcional, aspectos físicos, dor, estado geral de saúde e vitalidade; e na avaliação da EVA e do questionário RM.

No estudo realizado por Tavafian et al (2007) num Centro de Reumatologia no Irã, foram avaliados 102 pacientes, distribuídos aleatoriamente em dois grupos: grupo controle, o qual recebeu atendimento clínico, e grupo experimental, o qual participou de um programa de Escola de Postura. Os participantes foram avaliados a partir do questionário SF-36, o qual objetivou verificar a qualidade de vida nos dois grupos num período de três meses após finalizado o programa. Foi encontrado melhora significativa em todos as oito domínio do SF-36 para o grupo experimental.

Observa-se, portanto, que os resultados obtidos no presente estudo, em relação a melhora da qualidade de vida, foram efetivos como os resultados de programas de Escola da Postura verificados por Tsukimoto et al (2006) e Tavafian et al (2007), pois as médias de todos os domínios do questionário SF-36 melhoraram, e seis deles: capacidade funcional, limitações por aspectos físicos, dor, vitalidade, limitações emocionais e saúde mental obtiveram melhoras significativas.

Um dado considerado relevante, no presente estudo, foi o número de usuários que não aderiram à proposta do projeto de extensão Grupo de Coluna oferecido na UBS, os quais compareceram apenas na avaliação ou na avaliação e primeira aula. Ao verificar as características desses usuários, constatou-se que $100 \%$ eram do sexo feminino e que $38,89 \%$ dessas mulheres tinham 70 anos ou mais.

Pelas características destas pessoas, observadas na avaliação inicial ou na primeira aula, considera-se que as restrições de movimento relacionadas à idade que dificultam o deitar no chão para realização de alguns exercícios, a perda parcial da audição que impede acompanhar a parte teórica da aula e a dificuldade de deslocar-se até a UBS são motivos relevantes para a não adesão ao Grupo.

Outros autores (MACIEL, 2010; REICHERT et al., 2007) comentam a dificuldade de adesão a prática de atividades físicas em pessoas idosas, relacionando a não adesão desta população à falta de dinheiro, à sensação de cansaço e à falta de companhia. Satariano et al (2000) investigaram o processo de envelhecimento e a aptidão física em 2.046 indivíduos maiores de 55 anos, e comentam que entre as primeiras barreiras para a adesão à prática da atividade física nas mulheres são a falta de companhia e de interesse; a fadiga e os problemas de saúde. Nesse estudo, nas mulheres acima de 75 anos os problemas de saúde e funcionais, como medo de quedas, foram as principais barreiras mencionadas.

Constata-se, portanto, que, apesar dos usuários acima de 70 anos apresentarem dores musculoesqueléticas que requerem acompanhamento, o projeto de extensão Grupo de Coluna avaliado neste estudo não se mostrou 
eficiente para contemplar as necessidades desses usuários.

Em relação ao percentual de $50 \%$ encontrado na faixa etária abaixo de 60 anos que não aderiram ao Grupo, considera-se que o principal limitante seja o horário de oferecimento do projeto, o qual ocorre no turno da tarde e dificulta a participação daqueles que trabalham e a falta de interesse numa intervenção educativa.

No estudo de Andrade et al (2008) sobre a eficácia de um programa de Escola de Coluna, dos 70 participantes randomizados, 13 não completaram as avaliações. As causas para o abandono foram a não dispensa do trabalho, a mudança de endereço e a falta de interesse, as quais se aproximam as causas observadas neste estudo.

A faixa etária de maior adesão, no presente estudo, foi entre 60 e 69 anos, ou seja, de aposentados que ainda possuem uma boa mobilidade e autonomia. Em reunião com a equipe da UBS, onde foram apresentados os resultados do estudo, surgiu a proposta de oferecer Grupos de Coluna no final do turno da tarde, com o objetivo de viabilizar a participação de usuários trabalhadores e da necessidade de desenvolver uma proposta diferenciada para atingir os usuários acima de 70 anos.

\section{Considerações Finais}

Os Grupos de Coluna são programas de educação em saúde embasados nas Escolas Posturais que vem sendo utilizados como forma de prevenção e administração de dores musculoesqueléticas relacionadas a problemas cinético-funcionais nos serviços de atenção básica do SUS. Esses programas visam reduzir os quadros álgicos e contribuir para a melhora da qualidade de vida, desenvolvendo atividades teórico-práticas. São discutidos fatores relacionados à dor e à má postura e incentivadas à aquisição de posturas adequadas na execução de AVD's, além de exercícios para fortalecimento e alongamento, especialmente para coluna e membros inferiores.

A partir dos dados do presente estudo, concluiu-se que o Grupo de Coluna desenvolvido na UBS foi eficaz na diminuição da intensidade da dor, na melhora da funcionalidade e na melhora da qualidade de vida de seus usuários. Conclui-se também que uma intervenção educativa sobre os hábitos corporais e a realização de exercícios auxilia na redução de quadros de dores musculoesqueléticas e, portanto, devem ser considerados no processo de reabilitação e prevenção. Embora esta pesquisa tenha encontrado resultados positivos na finalização dos encontros, seria relevante dar continuidade a pesquisa, verificando a sua eficácia no médio e longo prazo e realizando um estudo com a inclusão de um grupo controle.

\section{Referências}

ALMEIDA, R. M.; CRISPIN, E. V. N.; MELO, V. M.; CARDIA, M. C. G. Análise comparativa entre diferentes meios terapêuticos nas algias da coluna vertebral. Revista Brasileira de Ciência e Saúde, João Pessoa, v. 3, n. 1, p. 29-36, 1999.

ANDRADE, S. C.; ARAÚJO, A. G. R.; VILAR, M. J. Escola de Coluna: revisão histórica e sua aplicação na lombalgia crônica. Revista Brasileira de Reumatologia, São Paulo, v. 45, n. 4, p. 224-228, 2005. Disponível em: $<$ http://dx.doi.org/10.1590/S048250042005000400006>. Acesso em: 17 set. 2010.

BRASIL. Ministério da Saúde. Secretaria de Vigilância em Saúde. Política Nacional de Promoção da Saúde. Brasília: Ministério da Saúde, 2006. (Série B. Textos Básicos de Saúde).

BRINGUENTE, M. E. O.; CASTRO, I. S.; JESUS, J. C. G.; LUCIANO, L. S. Fatores de risco para a coluna: avaliação em consulta de enfermagem. Revista Brasileira de Enfermagem, Brasília, v. 50, n. 3, p. 391-406, 1997.

BUSS, P. M. Promoção da saúde e qualidade de vida. Ciência \& Saúde Coletiva, Rio de Janeiro, v. 5 , n. 1, p. 163-177, 2000. Disponível em: $<$ http://redalyc.uaemex.mx/pdf/630/63050114.pdf> . Acesso em: 17 nov. 2010.

CANDEIAS, N. M. F. Conceitos de educação e de promoção em saúde: mudanças individuais e mudanças organizacionais. Revista de Saúde Pública, São Paulo, v. 31, n. 2, p. 209-213, 1997. Disponível em: $<$ http://dx.doi.org/10.1590/S0034$89101997000200016>$. Acesso em: 19 set. 2010.

CARAVIELLO, E. Z.; WASSERSTEIN, S.; CHAMLIAN, T. R.; MASIERO, D. Avaliação da dor e função de pacientes com lombalgia tratados com um programa de Escola de Coluna. Acta Fisiátrica, São Paulo, v. 12, n. 1, p. 11-14, 2005. Disponível em: $<$ http://www.actafisiatrica.org.br/v1\%5Ccontrole/se cure/Arquivos/AnexosArtigos/1C383CD30B7C298 AB50293ADFECB7B18/acta vol 12 num 0111 14.pdf>. Acesso em: 8 nov. 2010. 
CELICH, K. L. S.; GALON, C. Dor crônica em idosos e sua influência nas atividades da vida diária e convivência social. Revista Brasileira de Geriatria e Gerontologia, Rio de Janeiro, v. 12, n. 3, p. 345-359, 2009. Disponível em:

$<$ http://www.observatorionacionaldoidoso.fiocruz.b $\mathrm{r} /$ biblioteca/ artigos/144.pdf>. Acesso em: $12 \mathrm{mar}$. 2011.

CHUNG, T. M. Escola de coluna: experiência do Hospital das Clínicas da Universidade de São Paulo. Acta Fisiátrica, São Paulo, v. 3, n. 2, p. 13-17, 1996. Disponível em:

$<$ http://www.actafisiatrica.org.br/v1\%5Ccontrole/se cure/Arquivos/AnexosArtigos/045117B0E0A11A2 42B9765E79CBF113F/vl 03 n 02 13 17.pdf>.

Acesso em: 17 jun. 2010.

CICONELLI, R. M.; FERRAZ, M. B.; SANTOS, W. Tradução para a língua portuguesa e validação do questionário genérico de avaliação de qualidade de vida SF-36 (Brasil SF-36). Revista Brasileira de Reumatologia, São Paulo, v. 39, n. 3, p. 143150, 1998. Disponível em: $<$ http://www.nutrociencia.com.br/upload files/artig os download/qulalidade.pdf >. Acesso em: 31 maio 2011.

DEYO, R. A.; CHERKIN, D.; CONRAD, D.; VOLINN, E. Cost, controversy, crisis: low back pain and the health of the public. Annual Review of Public Health, Palo Alto, v. 12, p. 141-156, 1991. Disponível em:

$<$ http://dx.doi.org/10.1146/annurev.pu.12.050191. 001041>. Acesso em: 12 jan. 2011.

FAIRBANK, J. C.; COUPER, J.; DAVIES, J. B.; O'BRIEN, J. P. The oswestry low back pain disability questionnaire. Physiotherapy, Inglaterra, v. 66, n. 8, p. 271-273, 1980.

FAIRBANK, J. C. T.; PYNSENT, P. B. The oswestry disability index. Spine, Hagerstown, v. 25, n. 22, p. 2940-2952, 2000.

FERREIRA, M. S.; NAVEGA, M. T. Efeitos de um programa de orientação para adultos com lombalgia. Acta Ortopédica Brasileira, São Paulo, v. 18, n. 3, p. 127-131, 2010. Disponível em: <http://dx.doi.org/10.1590/S141378522010000300002>. Acesso em: 31 maio 2011.

FLECK, M. C.; SOUZA, J. C. R. P.; BARROS, N. H. S. A avaliação de qualidade de vida: guia para profissionais da saúde. Porto Alegre: Artmed, 2008.

FORSSELL, M. Z. The swedish back school. Physiotherapy, Inglaterra, v. 66, n. 4, p. 112-114, 1980.
FRYMOYER, J. W. E CATSBARIL, W. L. An overview of the Incidents and costs of low backback-pain. The Orthopedic Clinics of North America, Filadélfia, v. 22, n. 2, p. 263-271, 1991.

GAYA, A.; GARLIPP, D. C.; SILVA, M. F.; MOREIRA, R. B. Ciências do movimento humano: introdução à metodologia da pesquisa. Porto Alegre: Artmed, 2008.

GRIMLEY E. J. Prevention of age-associated loss of autonomy: epidemiological approaches.

Journal of Chronic Diseases, Inglaterra, v. 37, n. 5, p. 353-363, 1984. Disponível em: $<$ http://dx.doi.org/10.1016/0021-9681(84)901012>. Acesso em: 12 jan. 2011.

\section{KNOPLICH, J. Enfermidades da coluna}

vertebral: uma visão clínica e fisioterápica. 3. ed. São Paulo: Robe Editorial, 2003.

MACIEL, M. G. Atividade física e funcionalidade do idoso. Motriz, Rio Claro, v. 16, n. 4, p. 10241032, 2010. Disponível em: $<$ http://dx.doi.org/10.5016/19806574.2010v16n4p1024>. Acesso em: 12 jan. 2011.

MARTINEZ, M. C. As relações entre a satisfação com aspectos psicossociais no trabalho e a saúde do trabalhador. 2002. 255f. Dissertação (Mestrado) - Faculdade de Saúde Pública, Universidade de São Paulo, São Paulo, 2002. Disponível em:

$<$ http://www.teses.usp.br/teses/disponiveis/6/6134 /tde-07112006-210400/pt-br.php >. Acesso em 5 dez. 2010.

MAZO, G. Z. Atividade física, qualidade de vida e envelhecimento. Porto Alegre: Sulina, 2008.

OLIVEIRA, E. S.; GAZETTA, M. L. B.; SALIMENE, A. C. M. Dor crônica sob a ótica dos pacientes da escola de postura da DMR HC FMUSP. Acta Fisiátrica, São Paulo, v. 11, n. 1, p. 22-26, 2004. Disponível em: $<$ http://www.actafisiatrica.org.br/v1\%5Ccontrole/se cure/Arquivos/AnexosArtigos/C81E728D9D4C2F6 36F067F89CC14862C/artigo\%2003\%20acta v11 n01.pdf >. Acesso em: 13 fev. 2011.

REICHERT, F. F.; BARROS, A. J. D; DOMINGUES, M. R.; HALLAL, P. The role of perceived personal barriers to engagement in leisure-time physical activity. American Journal Public Health, Nova York, v. 97, n. 5, p. 515-519, 2007.

ROSA, T. E. C.; BENÍCIO, M. H. D.; LATORRE, M. R. D. O.; RAMOS, L. R. Fatores determinantes da capacidade funcional entre idosos. Revista de Saúde Pública, São Paulo, v. 37, n. 1, p. 40-48, 2003. Disponível em: 
$<$ http://dx.doi.org/10.1590/S0034$89102003000100008>$. Acesso em: 05 maio 2011.

SATARIANO, W. A.; HAIGHT, T. J.; TAGER, I. B. Reasons given by older people for limitation or avoidance of leisure time physical activity. Journal of the American Geriatrics Society, Nova York, v. 48, n. 5 , p. 505-512, 2000.

SCHMIDT, C. O.; KOHLMANN T. What do we know about the symptoms of back pain?: epidemiological results on prevalence, incidence, progression and risk factors. Zeitschrift für Orthopädie und ihre Grenzgebiete, Stuttgart, v. 143, n. 3, p. 292-298, 2005. Disponível em: <http://dx.doi.org/10.1055/s-2005-836631>. Acesso em: 5 dez. 2010.

SOUZA, J. L.; VIEIRA, A. Escola postural: um caminho para o conhecimento de si e o bem-estar corporal. Movimento, Porto Alegre, v. 9, n. 3, p. 101-122, 2003. Disponível em:

<http://seer.ufrgs.br/Movimento/article/view/2820/ 1435>. Acesso em: 19 jul. 2010.

TAVAFIAN, S. S.; JAMSHIDI, A.; KAZEM, M. K.; MONTAZERI, A. Low back pain education and short term quality of life: a randomized trial. BMC Musculoskeletal Disorders, Inglaterra, v. 8, p. 21, 2007. Disponível em: <http://dx.doi.org/10.1186/1471-2474-8-21>. Acesso em 8 nov. 2010.

TSUKIMOTO, G. R.; RIBEIRO, M.; BRITO, C. A.; BATISTELLA, L. R. Avaliação longitudinal da escola de postura para dor lombar crônica através da aplicação dos questionários Roland Morris e Short Form Health Survey (SF-36). Acta

Fisiátrica, São Paulo, v. 13, n. 2, p. 63-69, 2006. Disponível em:

$<$ http://www.actafisiatrica.org.br/v1/controle/secure /Arquivos/AnexosArtigos/DB8E1AF0CB3ACA1AE 2D0018624204529/editoracao vl 13 n 02 6369.pdf>. Acesso em: 28 jul. 2010.

VIEIRA, A. A Escola postural sob a perspectiva da educação somática: a reformulação de um programa de extensão na ESEF/UFRGS. 2004. Tese (Doutorado) - Universidade Federal do Rio Grande do Sul, Porto Alegre, 2004. Disponível em: <http://hdl.handle.net/10183/3942>. Acesso em: 13 fev. 2011.

VIGATTO, R. Adaptação cultural do instrumento "The Low Back Pain Disability Oswestry Questionnaire". 2006. Dissertação (Mestrado em Enfermagem) - Faculdade de Ciências Médicas, Universidade Estadual de Campinas, 2006.

Disponível em: $<$ http://www.bibliotecadigital.unicamp.br/document ? code $=000367690>$. Acesso em: 28 jul. 2010.
VIGATTO, R.; ALEXANDRE, N. M. C.; CORREA, $H$. R. Development of a brazilian portuguese version of the oswestry disability index: crosscultural adaptation, reliability, and validity. Spine, Hagerstown, v. 32, n. 4, p. 481-486, 2007. Disponível em: $<$ http://dx.doi.org/10.1097/01.brs.0000255075.114 96.47>. Acesso em: 8 nov. 2010.

Esse trabalho foi apresentado na Sessão Temática, Coordenação e Controle de Atividades Motoras e Métodos de Análise Biomecânica, no Congresso Internacional de Educação Física e Motricidade Humana 2011, promovido pela UNESP/Rio Claro.

Endereço:

Adriane Vieira

Universidade Federal do Rio Grande do Sul

Rua Felizardo, 750

Porto Alegre RS Brasil

e-mail: adriane.vieira@gmail.com

Recebido em: 28 de junho de 2011.

Aceito em: 1 de setembro de 2011.

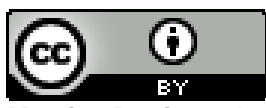

Motriz. Revista de Educação Física. UNESP, Rio Claro, SP, Brasil - elSSN: 1980-6574 - está licenciada sob Creative Commons - Atribuição 3.0 